\title{
Interactive comment on "Assessing the Added Value of the Intermediate Complexity Atmospheric Research Model (ICAR) for Precipitation in Complex Topography” by J. Horak et al.
}

\section{Anonymous Referee \#2}

Received and published: 5 February 2019

This manuscript assesses the added value of ICAR relative to coarse reanalysis for estimating precipitation in complex topography. Not yet widely evaluated, ICAR is a promising tool for a range of applications. The methods in this study are robust and the conclusions are important. The manuscript should be a valuable contribution to the literature.

\section{Comments}

1. In terms of the manuscript structure, it seems a bit unusual to have a combined "Methods and Results" section (4). I can see why the manuscript was structured as it is, but I wonder if it could be rationalised at all. Could there be benefits from a 
more "traditional" separation of methods and results? For example, the major sections could go something like: 1. Introduction 2. Study Area and Data 3. Methods 3.1

ICAR Overview and Setup 3.2 Evaluation Strategy 3.3 Skill Scores and Significance Tests 3.4 Flow Linearity (explaining how flow linearity and stability are calculated) 3.5 Weather Patterns (explaining dataset with figure of weather patterns) 4. Results (as currently structured but removing the methods now described in the previous section) 5. Discussion 6. Conclusions

This is just a possibility; there could be a better way. I would also suggest checking the manuscript for repetition and trying to minimise the amount of "referencing forward" (i.e. sometimes it is not necessary to say " $X$ will be discussed in Section $Y$ ").

2. One specific point on structure is that the optimal model top height is stated in Section 2.3, before the results from the sensitivity test are presented. This should be avoided I think. It is already stated in the relevant part of the results section (i.e. on model top sensitivity) that the remainder of the evaluation uses the optimal model top.

3. Also regarding the model top sensitivity, it would be interesting to contextualise the variation in ICAR performance shown in Figure 2 by providing the equivalent MSE for ERAI. This could be as a horizontal line on Figure 2 or just stated in the text. I.e., even for the model tops leading to larger errors, do they still outperform ERAI overall?

4. It is mentioned in the discussion (P29 L19-20) that higher model tops lead to lower precipitation. Does this apply across the full range of model tops tested? Would there be any value in adding a second panel to Figure 2 showing mean bias for the different model tops? I.e. given that ICAR is generally low-biased for the Alpine stations, does a $2.5 \mathrm{~km}$ model top lead to reduced bias (even if the MSE is little different from $4 \mathrm{~km}$ )? Or does ICAR become high-biased with a $2.5 \mathrm{~km}$ top?

Printer-friendly version

5. In several places in the manuscript, results are discussed but the corresponding figures/tables are "not shown". This includes the relationship between model top and precipitation magnitude mentioned above, as well as seasonal averages at Alpine sta-

Discussion paper 
tion locations (P16 L13-18). The latter case I found confusing initially, as the ERAI seasonality is reasonable-ish in Figure 5 but criticised in the text based on station locations (P16 L17-18). I would suggest considering whether some of the "not shown" figures/tables should be put into a supplement or whether references to them are necessary.

6. It may be useful to provide the season definitions used in Section 4.5 (i.e. which months).

7. The panels in Figure 5 are quite small so it is difficult to make out much of the detail. The overall improvement of ICAR over ERAI is clear though. It would be interesting to see a version of the figure zoomed in on the Alpine range, but perhaps this could be in future work.

\section{Figure 3b has a spelling error - "coastal".}

9. The boxplots for near-stable conditions in Figure 7c and 7d are quite different. What could be the reason for this?

10. There are a few places where the wording and grammar could be a little bit tighter. For example, sometimes "trend" is used when something like "pattern" might be better. There are other examples too, such as the first three sentences of the paragraph beginning on L15 on P20. The manuscript is generally fairly well written, but I would suggest that the authors check the wording and grammar throughout when making revisions.

11. In the abstract and discussion it is mentioned that ICAR can reduce MSE by up to $53 \%$. If this is the maximum reduction, what is the mean/median? This may be worth including to give the "overall" picture.

12. It could be mentioned again in the discussion/conclusion that a comparison of ICAR and WRF (or a similar model) might also be interesting for this study area. This might help us to understand some of the possible factors limiting ICAR performance discussed in Section 5. It would also give an idea of the relative performance gain from 
using WRF (if any) in a different climatic context to that tested in Gutmann et al. (2016).

Interactive comment on Hydrol. Earth Syst. Sci. Discuss., https://doi.org/10.5194/hess-2018HESSD 612, 2018.

Interactive

comment 